\title{
Certain expansion formulae of incomplete I-functions associated with the Leibniz rule
}

\author{
S. Meena ${ }^{a}$, S. BhatTeR ${ }^{a}$, K. JAnGid $^{b}$, S.D. Purohit ${ }^{b}, *$
}

a Department of Mathematics, Malaviya National Institute of Technology, Jaipur, India.

$\mathrm{b}$ Department of HEAS (Mathematics), Rajasthan Technical University, Kota, India.

- Received: 07.03.2021 • Accepted: 22.06.2021 • Published Online: 29.06.2021

\begin{abstract}
In this paper, we determine some expansion formulae of the incomplete I-functions in affiliation with the Leibniz rule for the Riemann-Liouville type derivatives. Further, expansion formulae of the incomplete $\overline{\mathrm{I}}$-function, incomplete $\overline{\mathrm{H}}$-function and incomplete $\mathrm{H}$-function are conferred as extraordinary instances of our primary outcomes.
\end{abstract}

Keywords: Incomplete Gamma functions, Incomplete I-functions, Fractional calculus, R-L fractional integral and derivative, Leibniz rule.

2010 MSC: 26A33, 33C20, 33C05..

\section{Introduction}

Rathie [1] described I-function in the following way:

$$
\begin{aligned}
\mathrm{I}_{\mathrm{p}, \mathrm{q}}^{\mathrm{m}, \mathrm{n}}[z] & =\mathrm{I}_{\mathrm{p}, \mathfrak{q}}^{\mathrm{m}, \mathrm{n}}\left[z \mid \begin{array}{c}
\left(\mathfrak{e}_{1}, v_{1} ; \mathcal{E}_{1}\right), \ldots,\left(\mathfrak{e}_{\mathfrak{p}}, v_{\mathfrak{p}} ; \mathcal{E}_{\mathfrak{p}}\right) \\
\left(\mathfrak{f}_{1}, \omega_{1} ; \mathcal{F}_{1}\right), \ldots,\left(\mathfrak{f}_{\mathfrak{p}}, \omega_{\mathfrak{p}} ; \mathcal{F}_{\mathfrak{p}}\right)
\end{array}\right] \\
& =\frac{1}{2 \pi \mathfrak{i}} \int_{\mathcal{L}} \psi(\mathrm{s}) z^{\mathrm{s}} \mathrm{ds},
\end{aligned}
$$

where,

$$
\psi(s)=\frac{\prod_{j=1}^{m}\left[\Gamma\left(\mathfrak{f}_{j}-\omega_{j} s\right)\right]^{\mathcal{F}_{j}} \prod_{j=1}^{n}\left[\Gamma\left(1-\mathfrak{e}_{j}+v_{j} s\right)\right]^{\varepsilon_{j}}}{\prod_{j=m+1}^{q}\left[\Gamma\left(1-\mathfrak{f}_{j}+\omega_{j} s\right)\right]^{\mathcal{F}_{j}} \prod_{j=n+1}^{p}\left[\Gamma\left(\mathfrak{e}_{j}-v_{j} s\right)\right]^{\varepsilon_{j}}},
$$


and $m, n, p, q \in N_{0}$ with $0 \leqslant n \leqslant p, 0 \leqslant m \leqslant q, v_{j}, \varepsilon_{j}(j=1, . ., p), \omega_{j}, \mathcal{F}_{j}(j=1, \ldots, q) \in$ $\mathbb{R}^{+}, \mathfrak{e}_{\mathfrak{j}}, \mathfrak{f}_{\mathfrak{j}} \in \mathbb{C}$. The suitable conditions for the $\mathcal{L}$ contour convergence depicted in (1.1) and portrayals just as the I-function information can be found in [1].

We next characterized the recognizable lower and upper incomplete gamma functions $\gamma(s, x)$ and $\Gamma(s, x)$, separately as:

$$
\gamma(s, x):=\int_{0}^{x} y^{s-1} e^{-y} d y, \quad(\Re(s)>0 ; x \geqq 0)
$$

and

$$
\Gamma(s, x):=\int_{x}^{\infty} y^{s-1} e^{-y} d y, \quad(x \geqq 0 ; \mathfrak{R}(s)>0 \quad \text { if } \quad x=0) .
$$

These functions satisfy the accompanying deterioration connection :

$$
\gamma(s, x)+\Gamma(s, x)=\Gamma(s)(\Re(s)>0) .
$$

Srivastava et al. [2] discovered a pair of Mellin-Barnes contour integral form of incomplete $\mathrm{H}$-functions $\gamma_{\mathrm{p}, \mathrm{q}}^{\mathrm{m}, \mathrm{n}}(z)$ and $\Gamma_{\mathrm{p}, \mathrm{q}}^{\mathrm{m}, \mathrm{n}}(z)$ and incomplete $\overline{\mathrm{H}}$-functions $\bar{\gamma}_{\mathrm{p}, \mathrm{q}}^{\mathrm{m}, \mathrm{n}}(z)$ and $\bar{\Gamma}_{\mathrm{p}, \mathrm{q}}^{\mathrm{m}, \mathrm{n}}(z)$ in terms of the $\gamma(s, x)$ and $\Gamma(s, x)$ represented by 1.3 and 1.4, respectively,

$$
\gamma_{\mathrm{p}, \mathrm{q}}^{\mathrm{m}, \mathrm{n}}(z)=\gamma_{\mathrm{p}, \mathrm{q}}^{\mathrm{m}, \mathrm{n}}\left[z \mid \begin{array}{c}
\left(\mathfrak{e}_{1}, v_{1}, x\right),\left(\mathfrak{e}_{j}, v_{j}\right)_{2, p} \\
\left(\mathfrak{f}_{j}, \omega_{j}\right)_{1, q}
\end{array}\right]=\frac{1}{2 \pi i} \int_{\mathcal{L}} g(s, x) z^{-s} \mathrm{ds}
$$

and

$$
\Gamma_{p, q}^{m, n}(z)=\Gamma_{p, q}^{m, n}\left[z \mid \begin{array}{c}
\left(\mathfrak{e}_{1}, v_{1}, x\right),\left(\mathfrak{e}_{j}, v_{j}\right)_{2, p} \\
\left(\mathfrak{f}_{j}, \omega_{j}\right)_{1, q}
\end{array}\right]=\frac{1}{2 \pi i} \int_{\mathcal{L}} \mathrm{G}(s, x) z^{-s} \mathrm{ds},
$$

where,

$$
g(s, x)=\frac{\gamma\left(1-\mathfrak{e}_{1}-v_{1} s, x\right) \prod_{j=1}^{m} \Gamma\left(\mathfrak{f}_{j}+\omega_{j} s\right) \prod_{j=2}^{n} \Gamma\left(1-\mathfrak{e}_{j}-v_{j} s\right)}{\prod_{j=m+1}^{q} \Gamma\left(1-\mathfrak{f}_{j}-\omega_{j} s\right) \prod_{j=n+1}^{p} \Gamma\left(\mathfrak{e}_{j}+v_{j} s\right)},
$$

and

$$
G(s, x)=\frac{\Gamma\left(1-\mathfrak{e}_{1}-v_{1} s, x\right) \prod_{j=1}^{m} \Gamma\left(\mathfrak{f}_{j}+\omega_{j} s\right) \prod_{j=2}^{n} \Gamma\left(1-\mathfrak{e}_{j}-v_{j} s\right)}{\prod_{j=m+1}^{q} \Gamma\left(1-\mathfrak{f}_{j}-\omega_{j} s\right) \prod_{j=n+1}^{p} \Gamma\left(\mathfrak{e}_{j}+v_{j} s\right)},
$$

with the arrangement of conditions setout in [2].

These incomplete $\mathrm{H}$-functions satisfy the accompanying deterioration connection:

$$
\gamma_{p, q}^{m, n}(z)+\Gamma_{p, q}^{m, n}(z)=H_{p, q}^{m, n}(z) .
$$

The incomplete H-functions $\gamma_{p, q}^{m, n}(z)$ and $\Gamma_{p, q}^{m, n}(z)$ described in (1.6) and (1.7) exist for $x \geqslant 0$, under the set of conditions given by Srivastava et al. [2]. 
Jangid et al. [3] presented a group of the incomplete I-functions ${ }^{\gamma} \operatorname{I}_{\mathfrak{p}, q}^{m}(z)$ and ${ }^{\Gamma} I_{p, q}^{m, n}(z)$ which prompts a characteristic speculation and disintegration equation for I-function:

$$
\begin{aligned}
\gamma_{\mathrm{p}, \mathrm{q}}^{\mathrm{m}, \mathrm{n}}[z] & =\gamma_{\mathrm{I}_{\mathrm{p}, \mathrm{q}}^{\mathrm{m}, \mathrm{n}}}\left[z \mid \begin{array}{c}
\left(\mathfrak{e}_{1}, v_{1} ; \mathcal{E}_{1}: x\right),\left(\mathfrak{e}_{2}, v_{2} ; \mathcal{E}_{2}\right), \ldots,\left(\mathfrak{e}_{\mathfrak{p}}, v_{\mathrm{p}} ; \mathcal{E}_{\mathrm{p}}\right) \\
\left(\mathfrak{f}_{1}, \omega_{1} ; \mathcal{F}_{1}\right),\left(\mathfrak{f}_{2}, \omega_{2} ; \mathcal{F}_{2}\right), \ldots,\left(\mathfrak{f}_{\mathrm{q}}, \omega_{\mathrm{q}} ; \mathcal{F}_{\mathrm{q}}\right)
\end{array}\right] \\
& =\frac{1}{2 \pi \mathrm{i}} \int_{\mathcal{L}} \phi(s, x) z^{\mathrm{s}} \mathrm{ds},
\end{aligned}
$$

and

$$
\begin{aligned}
\Gamma_{\mathrm{I}_{\mathrm{p}, \mathrm{q}}^{\mathrm{m}, \mathrm{n}}[z]} & =\Gamma_{\mathrm{I}_{\mathrm{p}, \mathrm{q}}^{\mathrm{m}, \mathrm{n}}}\left[z \mid \begin{array}{c}
\left(\mathfrak{e}_{1}, v_{1} ; \mathcal{E}_{1}: x\right),\left(\mathfrak{e}_{2}, v_{2} ; \mathcal{E}_{2}\right), \ldots,\left(\mathfrak{e}_{\mathrm{p}}, v_{\mathrm{p}} ; \mathcal{E}_{\mathrm{p}}\right) \\
\left(\mathfrak{f}_{1}, \omega_{1} ; \mathcal{F}_{1}\right),\left(\mathfrak{f}_{2}, \omega_{2} ; \mathcal{F}_{2}\right), \ldots,\left(\mathfrak{f}_{\mathrm{q}}, \omega_{\mathrm{q}} ; \mathcal{F}_{\mathrm{q}}\right)
\end{array}\right] \\
& =\frac{1}{2 \pi \mathrm{i}} \int_{\mathcal{L}} \Phi(\mathrm{s}, \mathrm{x}) z^{\mathrm{s}} \mathrm{ds},
\end{aligned}
$$

for all $z \neq 0$, where,

$$
\phi(s, x)=\frac{\left[\gamma\left(1-\mathfrak{e}_{1}+v_{1} s, x\right)\right]^{\mathcal{E}_{1}} \prod_{j=1}^{m}\left[\Gamma\left(\mathfrak{f}_{j}-\omega_{j} s\right)\right]^{\mathcal{F}_{j}} \prod_{j=2}^{n}\left[\Gamma\left(1-\mathfrak{e}_{j}+v_{j} s\right)\right]^{\varepsilon_{j}}}{\prod_{j=m+1}^{q}\left[\Gamma\left(1-\mathfrak{f}_{j}+\omega_{j} s\right)\right]^{\mathcal{F}_{j}} \prod_{j=n+1}^{p}\left[\Gamma\left(\mathfrak{e}_{j}-v_{j} s\right)\right]^{\mathcal{E}_{j}}},
$$

and

$$
\Phi(s, x)=\frac{\left[\Gamma\left(1-\mathfrak{e}_{1}+v_{1} s, x\right)\right]^{\varepsilon_{1}} \prod_{j=1}^{m}\left[\Gamma\left(\mathfrak{f}_{j}-\omega_{j} s\right)\right]^{\mathcal{F}_{j}} \prod_{j=2}^{n}\left[\Gamma\left(1-\mathfrak{e}_{j}+v_{j} s\right)\right] \varepsilon_{j}}{\prod_{j=m+1}^{\mathrm{q}}\left[\Gamma\left(1-\mathfrak{f}_{j}+\omega_{j} s\right)\right]^{\mathcal{F}_{j}} \prod_{j=\mathfrak{n}+1}^{p}\left[\Gamma\left(\mathfrak{e}_{j}-v_{j} s\right)\right]^{\varepsilon_{j}}} .
$$

The incomplete I-functions $\gamma \mathrm{I}_{\mathfrak{p}, \mathfrak{q}}^{m, n}[z]$ and $\Gamma_{\mathrm{I}_{\mathfrak{p}, \mathfrak{q}}^{m, \mathfrak{n}}}[z]$ in 1.11 and 1.12 exist for all $x \geqslant 0$ under indistinguishable form and conditions from expressed in Rathie [1]. For $\mathcal{E}_{1}=1$, the definitions 1.11 and 1.12 at once yield the following division relation:

$$
\gamma_{\mathrm{I}_{\mathbf{p}, \mathbf{q}}^{\mathrm{m}} \mathrm{n}}^{\mathrm{n}}[z]+\Gamma_{\mathrm{p}, \mathbf{q}}^{\mathrm{m}, \mathrm{n}}[z]=\mathrm{I}_{\mathbf{p}, \mathbf{q}}^{\mathrm{m}, \mathrm{n}}[z] \quad\left(\varepsilon_{1}=1\right),
$$

for the familiar I-function.

The incomplete I-functions ${ }^{\gamma} \mathrm{I}_{\mathfrak{p}, \mathfrak{q}}^{\mathrm{m}, \mathfrak{n}}[z]$ and $\Gamma_{\mathrm{p}, \mathfrak{q}}^{\mathrm{m}, \mathfrak{n}}[z]$ defined in 1.11 and 1.12 exist for all $x \geqslant 0$, under the set of conditions given by Rathie [1], with

$$
\Delta>0,|\arg z|<\Delta \frac{\pi}{2}
$$

where,

$$
\Delta=\sum_{j=1}^{m} \mathcal{F}_{j} \omega_{j}-\sum_{j=m+1}^{q} \mathcal{F}_{j} \omega_{j}+\sum_{j=1}^{n} \varepsilon_{j} v_{j}-\sum_{j=n+1}^{p} \varepsilon_{j} v_{j} .
$$

Numerous authors are currently working on variety applications of these incomplete functions. Recently, Bansal et al. [4, 5, 6, 7] discussed about some of the new outcomes 
and uses of the incomplete $\mathrm{H}$-functions; such as they solved the Fredholm-type integral equation involving incomplete $\mathrm{H}$-function and incomplete $\overline{\mathrm{H}}$-function in the kernel, useful classical integral transforms of the $\aleph$-functions, some new outcomes of Srivastava-LuoRaina $\mathrm{M}$-transform associated to incomplete $\mathrm{H}$-function, and Oberhettinger's integral formula involving $M$-series. Recent studies $[8,9,10,11]$ for further details on the characteristics of incomplete I-functions.

In $[12,13,14]$, the authors discussed an extension of the generalized Mittag-leffler function in terms of the generalized Pochhammer symbol, determined some results of the generalized Bessel-Maitland function in the field of the fractional calculus and new extention of Caputo fractional derivative operator.

The Riemann-Liouville fractional operators of order $v$ for $f(z)$ are characterized as follows (see [15]):

$$
I^{v} f(z)=\frac{1}{\Gamma(v)} \int_{0}^{z}(z-t)^{v-1} f(t) d t,
$$

here, the integral is all around characterized gave $f$ is a locally integrable function and $v$ is a complex number in the half plane $\mathfrak{R}(v)>0$.

$$
\mathrm{D}_{z}^{v} \mathrm{f}(z)=\frac{1}{\Gamma(-v)} \int_{0}^{z}(z-t)^{(-v-1)} f(t) d t(\Re(v)<0),
$$

If $\mathfrak{R}(v) \geqslant 0$ and $m \in \mathbb{N}$ is the smallest integer with $m-1 \leqslant \mathfrak{R}(v)<\mathrm{m}$, then

$$
D_{z}^{v} f(z)=\frac{d^{m}}{d z^{m}} D_{z}^{v-m} f(z)==\frac{d^{m}}{d z^{m}}\left[\frac{1}{\Gamma(-v+m)} \int_{0}^{z}(z-t)^{-v+m-1} f(t) d t\right] .
$$

The classical Leibniz rule for two differentiable functions $f$ and $g$ is defined as follows:

$$
D^{n}[f(t) g(t)]=\sum_{k=0}^{n}\left(\begin{array}{l}
n \\
k
\end{array}\right)\left[D^{k} g(t)\right]\left[D^{n-k} f(t)\right] .
$$

This Leibniz rule can be reached out for the Riemann-Liouville type derivatives. On the off chance that $f$ and $g$ are two functions of class $C$, at that point the fractional speculation of the Leibniz rule is characterized as (see [16])

$$
D^{\mu}[f(t) g(t)]=\sum_{k=0}^{\infty}\left(\begin{array}{l}
\mu \\
k
\end{array}\right)\left[D^{k} g(t)\right]\left[D^{\mu-k} f(t)\right] ; \mu>0, \quad k \in \mathbb{N} .
$$

In particular, if $f$ is function of class $C$, then

$$
D^{\mu}\left[t^{p} f(t)\right]=\sum_{r=0}^{p}\left(\begin{array}{l}
\mu \\
r
\end{array}\right)\left[D^{r} t^{p}\right]\left[D^{\mu-r} f(t)\right], \quad \mu>0 .
$$

The Leibniz rule, which sums up the differentiation law of the product, might be utilized to extricate an instrument that ascertains the organization portrayal of the differential operators. 


\section{Main Results}

We establish some expansion formulae of the incomplete I-functions by the use of the Leibniz rule.

Theorem 2.1. Let $\lambda \geqslant 1, \sigma>0, k \in \mathbb{N}, m \in \mathbb{N}$ and $m-1 \leqslant \mathfrak{R}(\mu) \leqslant m$, then the following result holds:

$$
\begin{aligned}
& \left.\Gamma_{I_{p+1, q+1}^{m, n+1}}^{m a z^{\sigma}} \begin{array}{c}
\left(\mathfrak{e}_{1}, v_{1} ; \mathcal{E}_{1}: x\right),(1-\lambda, \sigma ; 1),\left(\mathfrak{e}_{j}, v_{j} ; \mathcal{E}_{j}\right)_{2, p} \\
\left(\mathfrak{f}_{j}, \omega_{j} ; \mathcal{F}_{j}\right)_{1, q},(1-\lambda+\mu, \sigma ; 1)
\end{array}\right] \\
& =\sum_{k=0}^{\infty}\left(\begin{array}{l}
\mu \\
k
\end{array}\right) \frac{\Gamma(\lambda)}{\Gamma(\lambda-\mu+k)} \Gamma_{p+1, q+1}^{m, n+1}\left[a z^{\sigma} \mid \begin{array}{c}
\left(\mathfrak{e}_{1}, v_{1} ; \mathcal{E}_{1}: x\right),(0, \sigma ; 1),\left(\mathfrak{e}_{j}, v_{j} ; \mathcal{E}_{j}\right)_{2, p} \\
\left(\mathfrak{f}_{j}, \omega_{j} ; \mathcal{F}_{j}\right)_{1, q},(k, \sigma ; 1)
\end{array}\right] .
\end{aligned}
$$

Proof. To prove the result 2.1 , let us consider $\mathrm{f}(z)=z^{\lambda-1}$ and

$$
g(z)=\Gamma_{\mathfrak{p}, \mathfrak{q}}^{m, n}\left[a z^{\sigma}\right]=\Gamma_{\mathrm{I}_{\mathrm{p}, \mathrm{q}} \mathrm{m}, \mathrm{n}}\left[\mathrm{a} z^{\sigma} \mid \begin{array}{c}
\left(\mathfrak{e}_{1}, v_{1} ; \mathcal{E}_{1}: x\right),\left(\mathfrak{e}_{j}, v_{j} ; \mathcal{E}_{j}\right)_{2, p} \\
\left(\mathfrak{f}_{j}, \omega_{j} ; \mathcal{F}_{j}\right)_{1, q}
\end{array}\right] .
$$

Now substituting the values of $f(z)$ and $g(z)$ in 1.19 , we obtain

$$
\mathrm{D}^{\mu}\left[z^{\lambda-1} \Gamma_{\mathrm{p}, \mathrm{q}}^{\mathrm{m}, \mathrm{n}}\left(a z^{\sigma}\right)\right]=\sum_{\mathrm{k}=0}^{\infty}\left(\begin{array}{l}
\mu \\
k
\end{array}\right)\left[\mathrm{D}^{\mathrm{k} \Gamma} \mathrm{I}_{\mathrm{p}, \mathrm{q}}^{\mathrm{m}, \mathrm{n}}\left(\mathrm{a} z^{\sigma}\right)\right]\left[\mathrm{D}^{\mu-k} z^{\lambda-1}\right] .
$$

On taking L.H.S of equation 2.2, we obtain

$$
\begin{aligned}
\mathrm{D}^{\mu}\left[z^{\lambda-1} \Gamma_{\mathrm{p}, \mathrm{q}}^{\mathrm{m}, \mathrm{n}}\left(a z^{\sigma}\right)\right] & =\mathrm{D}^{\mu}\left[z^{\lambda-1} \frac{1}{2 \pi \mathrm{i}} \int_{\mathcal{L}} \Phi(s, x) \mathrm{a}^{s} z^{\sigma s} \mathrm{~d} s\right] \\
& =\frac{1}{2 \pi i} \int_{\mathcal{L}} \Phi(s, x) \mathrm{a}^{s} \mathrm{D}^{\mu}\left[z^{\lambda+\sigma s-1}\right] \mathrm{d} s \\
& =\frac{1}{2 \pi i} \int_{\mathcal{L}} \Phi(s, x) \mathrm{a}^{-s} \frac{\Gamma(\lambda+\sigma s)}{\Gamma(\lambda-\mu+\sigma s)} z^{\lambda-\mu+\sigma s-1} \mathrm{~d} s .
\end{aligned}
$$

In view of the definition 1.12 , we have

$$
\begin{aligned}
& \mathrm{D}^{\mu}\left[z^{\lambda-1} \Gamma_{\mathrm{p}, \mathrm{q}}^{\mathrm{m}, \mathrm{n}}\left(\mathrm{a} z^{\sigma}\right)\right]=z^{\lambda-\mu-1} \times \\
& \Gamma_{I_{\mathfrak{p}+1, q+1}^{m, n+1}}\left[a z^{\sigma} \mid \begin{array}{c}
\left(\mathfrak{e}_{1}, v_{1} ; \mathcal{E}_{1}: x\right),(1-\lambda, \sigma ; 1),\left(\mathfrak{e}_{\mathfrak{j}}, v_{j} ; \mathcal{E}_{\mathfrak{j}}\right)_{2, p} \\
\left(\mathfrak{f}_{j}, \omega_{j} ; \mathcal{F}_{\mathfrak{j}}\right)_{1, q},(1-\lambda+\mu, \sigma ; 1)
\end{array}\right] .
\end{aligned}
$$

Similarly, the R.H.S. of equation 2.2 is the immediate consequences of the definitions 1.12 and 1.19 , we obtain

$$
\begin{aligned}
& \sum_{k=0}^{\infty}\left(\begin{array}{l}
\mu \\
k
\end{array}\right)\left[D^{k} \Gamma I_{p, q}^{m, n}\left(a z^{\sigma}\right)\right]\left[D^{\mu-k} z^{\lambda-1}\right] \\
& =z^{\lambda-\mu-1} \sum_{k=0}^{\infty}\left(\begin{array}{l}
\mu \\
k
\end{array}\right) \frac{\Gamma(\lambda)}{\Gamma(\lambda-\mu+k)} \Gamma_{p+1, q+1}^{m, n+1}\left[\begin{array}{c}
a z^{\sigma} \\
\left(\mathfrak{e}_{1}, v_{1} ; \mathcal{E}_{1}: x\right),(0, \sigma),\left(\mathfrak{e}_{j}, v_{j} ; \mathcal{E}_{j}\right)_{2, p} \\
\left(\mathfrak{f}_{j}, \omega j ; \mathcal{F}_{j}\right)_{1, q},(k, \sigma ; 1)
\end{array}\right] .
\end{aligned}
$$

Substituting the equation 2.3 and 2.4 into 2.2 , we get the required result 2.1. 
Below theorem are the immediate consequences of the definitions 1.11, 1.12 and 1.19 and hence they are given without proof here.

Theorem 2.2. Let $\lambda \geqslant 1, \sigma>0, k \in \mathbb{N}, m \in \mathbb{N}$ and $m-1 \leqslant \mathfrak{R}(\mu) \leqslant m$, then the following result holds:

$$
\begin{aligned}
& \Gamma_{\mathrm{I}_{\mathrm{p}+1, \mathrm{q}+1}^{\mathrm{m}, \mathrm{n}+1}}\left[\mathrm{a} z^{-\sigma} \mid \begin{array}{c}
\left(\mathfrak{e}_{1}, v_{1} ; \mathcal{E}_{1}: x\right),\left(\mathfrak{e}_{j}, v_{j} ; \mathcal{E}_{\mathbf{j}}\right)_{2, \mathrm{p}},(\lambda-\mu, \sigma ; 1) \\
(\lambda, \sigma ; 1),\left(\mathfrak{f}_{j}, \omega_{j} ; \mathcal{F}_{j}\right)_{1, q}
\end{array}\right] \\
& =\sum_{\mathrm{k}=0}^{\infty}\left(\begin{array}{l}
\mu \\
k
\end{array}\right) \frac{\Gamma(\lambda)}{\Gamma(\lambda-\mu+k)} \Gamma_{\mathrm{p}+1, \mathbf{q}+1}^{\mathrm{m}, \mathrm{n}+1}\left[\begin{array}{c|c}
\mathrm{a} z^{-\sigma} & \left(\mathfrak{e}_{1}, v_{1} ; \mathcal{E}_{1}: x\right),\left(\mathfrak{e}_{j}, \boldsymbol{v}_{j} ; \mathcal{E}_{\mathfrak{j}}\right)_{2, p},(1-k, \sigma ; 1) \\
(1, \sigma ; 1),\left(\mathfrak{f}_{j}, \omega_{j} ; \mathfrak{F}_{j}\right)_{1, q}
\end{array}\right] .
\end{aligned}
$$

Theorem 2.3. Let $\lambda \geqslant 1, \sigma>0, k \in \mathbb{N}, m \in \mathbb{N}$ and $m-1 \leqslant \mathfrak{R}(\mu) \leqslant m$, then the following result holds:

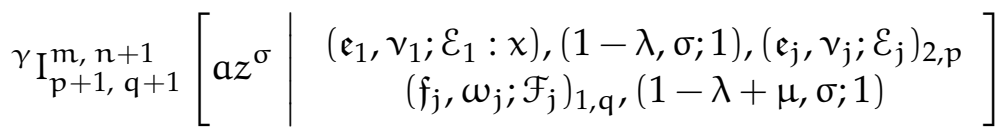

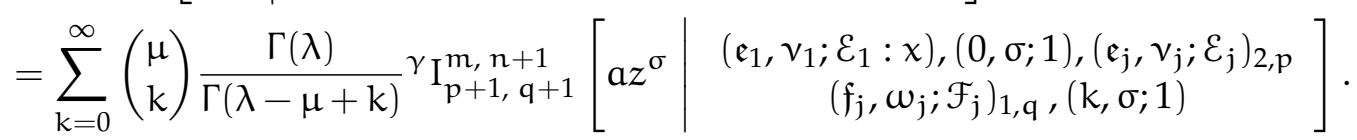

Theorem 2.4. Let $\lambda \geqslant 1, \sigma>0, k \in \mathbb{N}, m \in \mathbb{N}$ and $m-1 \leqslant \mathfrak{R}(\mu) \leqslant m$, then the following result holds:

$$
\begin{aligned}
& \gamma_{\mathrm{p}+1, \mathbf{q}+1}^{\mathrm{m}, \mathrm{n}+1}\left[\mathrm{a} z^{-\sigma} \mid \begin{array}{c}
\left(\mathfrak{e}_{1}, v_{1} ; \mathcal{E}_{1}: x\right),\left(\mathfrak{e}_{\mathfrak{j}}, \boldsymbol{v}_{\mathbf{j}} ; \mathcal{E}_{\mathbf{j}}\right)_{2, \mathrm{p}},(\lambda-\mu, \sigma ; 1) \\
(\lambda, \sigma ; 1),\left(\mathfrak{f}_{j}, \omega_{j} ; \mathcal{F}_{j}\right)_{1, q}
\end{array}\right] \\
& =\sum_{k=0}^{\infty}\left(\begin{array}{l}
\mu \\
k
\end{array}\right) \frac{\Gamma(\lambda)}{\Gamma(\lambda-\mu+k)} \gamma_{p+1, q+1}^{m, n+1}\left[\begin{array}{c}
a z^{-\sigma} \\
\left(\mathfrak{e}_{1}, v_{1} ; \mathcal{E}_{1}: x\right),\left(\mathfrak{e}_{j}, v_{j} ; \mathcal{E}_{j}\right)_{2, p},(1-k, \sigma ; 1), \\
(1, \sigma ; 1),\left(\mathfrak{f}_{j}, \omega_{j} ; \mathcal{F}_{j}\right)_{1, q}
\end{array}\right] .
\end{aligned}
$$

\section{Special Cases}

In this section, we determine a few expansion formulae for the incomplete $\overline{\mathrm{I}}$-function, incomplete $\overline{\mathrm{H}}$-function and incomplete $\mathrm{H}$-function, as special instances of Theorem 2.1 and Theorem 2.2. For the meanings of incomplete $\overline{\mathrm{H}}$-function and incomplete $\mathrm{H}$-function one could see Srivastava [2]. To delineate the uses of fundamental outcomes, if we give the specific qualities to the parameters, then we obtain the following special cases:

(i) Incomplete $\bar{I}$-function: If we set $\mathcal{F}_{j}=1(j=1, \cdots, m)$ in 1.12 and utilizing the connection, that is

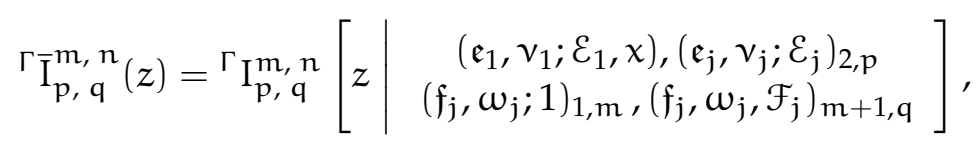

in 2.1 and 2.5, respectively, at that point we get the accompanying corollaries: 
Corollary 3.1. Let $\lambda \geqslant 1, k \in \mathbb{N}, m \in \mathbb{N}$ and $m-1 \leqslant \mathfrak{R}(\mu) \leqslant m$, then the following formula holds:

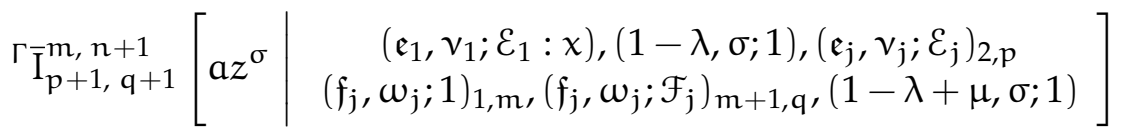

$$
\begin{aligned}
& =\sum_{k=0}^{\infty}\left(\begin{array}{l}
\mu \\
k
\end{array}\right) \frac{\Gamma(\lambda)}{\Gamma(\lambda-\mu+k)} \Gamma_{\bar{I}_{p+1, q}}^{m, n+1}\left[a z^{\sigma} \mid \begin{array}{c}
\left(\mathfrak{e}_{1}, v_{1} ; \mathcal{E}_{1}: x\right),(0, \sigma ; 1),\left(\mathfrak{e}_{j}, v_{j} ; \mathcal{E}_{j}\right)_{2, p} \\
\left(\mathfrak{f}_{j}, \omega_{j} ; 1\right)_{1, \mathfrak{m}},\left(\mathfrak{f}_{j}, \omega_{j} ; \mathcal{F}_{j}\right)_{\mathfrak{m}+1, q},(k, \sigma ; 1)
\end{array}\right] .
\end{aligned}
$$

Corollary 3.2. Let $\lambda \geqslant 1, k \in \mathbb{N}, m \in \mathbb{N}$ and $m-1 \leqslant \Re(\mu) \leqslant m$, then the following result holds:

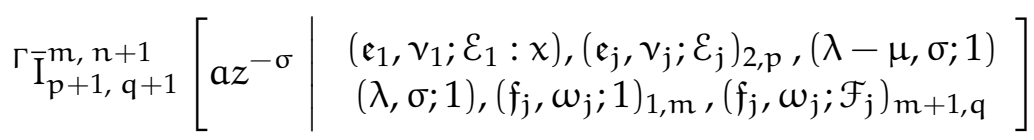

$$
\begin{aligned}
& =\sum_{k=0}^{\infty}\left(\begin{array}{l}
\mu \\
k
\end{array}\right) \frac{\Gamma(\lambda)}{\Gamma(\lambda-\mu+k)} \Gamma_{p+1, q+1}^{m, n+1}\left[\begin{array}{l|l}
a z^{-\sigma} & \begin{array}{l}
\left(\mathfrak{e}_{1}, v_{1} ; \mathcal{E}_{1}: x\right),\left(\mathfrak{e}_{j}, v_{j} ; \mathcal{E}_{j}\right)_{2, p},(1-k, \sigma ; 1) \\
(1, \sigma ; 1),\left(\mathfrak{f}_{j}, \omega_{j} ; 1\right)_{1, m},\left(\mathfrak{f}_{j}, \omega_{j} ; \mathcal{F}_{j}\right)_{m+1, q}
\end{array}
\end{array}\right] .
\end{aligned}
$$

(ii) Incomplete $\overline{\mathrm{H}}$-function $\bar{\Gamma}_{\mathrm{p}, \mathrm{q}}^{\mathrm{m}, \mathrm{n}}(z)$ : if we give particular values to the parameters, such as $\mathcal{F}_{j}(j=1, \cdots, \mathfrak{m})=1$ and $\mathcal{E}_{j}(j=n+1, \cdots, p)=1$ in 1.12 , and using the below relation (see [2])

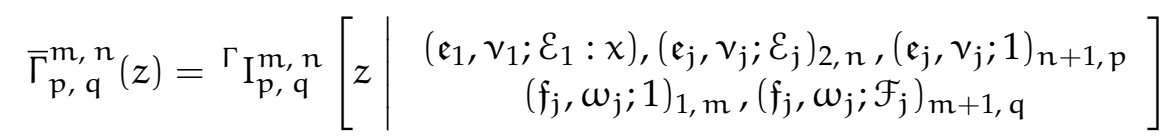

$$
\begin{aligned}
& =\bar{\Gamma}_{\mathrm{p}, \mathrm{q}}^{\mathrm{m}, \mathrm{n}}\left[\boldsymbol{z} \mid \begin{array}{c}
\left(\mathfrak{e}_{1}, v_{1} ; \mathcal{E}_{1}: x\right),\left(\mathfrak{e}_{j}, v_{j} ; \mathcal{E}_{j}\right)_{2, n},\left(\mathfrak{e}_{j}, v_{\mathfrak{j}}\right)_{\mathfrak{n}+1, p} \\
\left(\mathfrak{f}_{j}, \omega_{j}\right)_{1, m},\left(\mathfrak{f}_{j}, \omega_{j} ; \mathcal{F}_{j}\right)_{m+1, q}
\end{array}\right],
\end{aligned}
$$

we get the following corollaries:

Corollary 3.3. Let $\lambda \geqslant 1, \sigma>0, k \in \mathbb{N}, m \in \mathbb{N}$ and $m-1 \leqslant \mathfrak{R}(\mu) \leqslant m$, then the following result holds:

$$
\begin{aligned}
& \bar{\Gamma}_{p+1, q+1}^{m, n+1}\left[a z^{\sigma} \mid \begin{array}{c}
\left(\mathfrak{e}_{1}, v_{1} ; \mathcal{E}_{1}: x\right),(1-\lambda, \sigma ; 1),\left(\mathfrak{e}_{j}, v_{j} ; \mathcal{E}_{j}\right)_{2, n}, \\
\left(\mathfrak{f}_{j}, \omega_{j}\right)_{1, m},\left(\mathfrak{f}_{j}, \omega_{j} ; \mathcal{F}_{j}\right)_{m+1, q},(1-\lambda+\mu, \sigma ; 1)
\end{array}\right] \\
& =\sum_{k=0}^{\infty}\left(\begin{array}{l}
\mu \\
k
\end{array}\right) \frac{\Gamma(\lambda)}{\Gamma(\lambda-\mu+k)} \times \\
& \bar{\Gamma}_{p+1, q+1}^{m, n+1}\left[a z^{\sigma} \mid \begin{array}{c}
\left(\mathfrak{e}_{1}, v_{1} ; \mathcal{E}_{1}: x\right),(0, \sigma ; 1),\left(\mathfrak{e}_{j}, v_{j} ; \mathcal{E}_{j}\right)_{2, n},\left(\mathfrak{e}_{j}, v_{j}\right)_{n+1, p} \\
\left(\mathfrak{f}_{j}, \omega_{j}\right)_{1, m},\left(\mathfrak{f}_{j}, \omega_{j} ; \mathcal{F}_{j}\right)_{m+1, q},(k, \sigma ; 1)
\end{array}\right] .
\end{aligned}
$$


Corollary 3.4. Let $\lambda \geqslant 1, \sigma>0, k \in \mathbb{N}, m \in \mathbb{N}$ and $m-1 \leqslant \mathfrak{R}(\mu) \leqslant m$, then the following result holds:

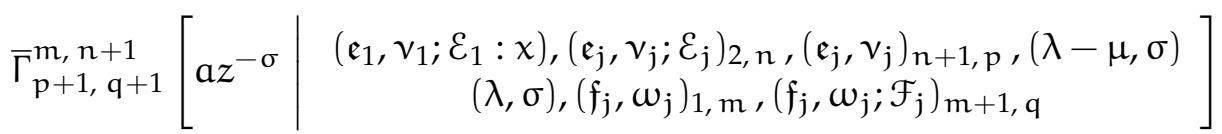

$$
\begin{aligned}
& =\sum_{k=0}^{\infty}\left(\begin{array}{l}
\mu \\
k
\end{array}\right) \frac{\Gamma(\lambda)}{\Gamma(\lambda-\mu+k)} \times \\
& \bar{\Gamma}_{p+1, q+1}^{m, n+1}\left[a z^{-\sigma} \mid \begin{array}{c}
\left(\mathfrak{e}_{1}, v_{1} ; \mathcal{E}_{1}: x\right),\left(\mathfrak{e}_{j}, v_{j} ; \mathcal{E}_{\mathfrak{j}}\right)_{2, n},\left(\mathfrak{e}_{j}, v_{j}\right)_{n+1, p},(1-k, \sigma) \\
(1, \sigma),\left(\mathfrak{f}_{j}, \omega_{j}\right)_{1, m},\left(\mathfrak{f}_{j}, \omega_{j} ; \mathcal{F}_{j}\right)_{m+1, q}
\end{array}\right] .
\end{aligned}
$$

(iii) Incomplete $H$-function $\Gamma_{p, q}^{m, n}(z)$ : if we put $\mathcal{F}_{j}(j=1, \cdots, q)=1$ and $\mathcal{E}_{j}(j=$ $1, \cdots, p)=1$ in 1.12 , and utilizing the connection, that is (see [2])

$$
\begin{aligned}
& \Gamma_{\mathfrak{p}, \mathfrak{q}}^{m, n}(z)=\Gamma_{I_{p}, \mathfrak{q}}^{m, n}\left[z \mid \begin{array}{c}
\left(\mathfrak{e}_{1}, v_{1} ; 1: x\right),\left(\mathfrak{e}_{j}, v_{j} ; 1\right)_{2, p} \\
\left(\mathfrak{f}_{j}, \omega_{j} ; 1\right)_{1, q}
\end{array}\right] \\
& =\Gamma_{\mathfrak{p}, \mathfrak{q}}^{m, n}\left[z \mid \begin{array}{c}
\left(\mathfrak{e}_{1}, v_{1}: x\right),\left(\mathfrak{e}_{\mathfrak{j}}, v_{\mathfrak{j}}\right)_{2, \mathfrak{p}} \\
\left(\mathfrak{f}_{j}, \omega_{\mathfrak{j}}\right)_{1, q}
\end{array}\right],
\end{aligned}
$$

in 2.1 and 2.5 respectively, then we get the results obtained by Meena et al. [17, Theorem 2.1. and Theorem 2.2.].

Remark: Similarly, special cases for the Theorem 2.3 and Theorem 2.4 may be derived.

\section{Conclusion}

In this paper, by making use of the Leibniz rule for the Riemann-Liouville type derivatives, we have obtained certain expansion formulae of the incomplete I-functions. For our main findings, we have discussed some special cases and some known results also appeared.

\section{Acknowledgement}

The authors thank the referees for their concrete suggestions which resulted in a better organization of this article.

\section{References}

[1] Rathie AK (1997). A new generalization of generalized Hypergeometric functions. Le Math. LII: 297-310.

[2] Srivastava HM, Saxena RK and Parmar RK (2018). Some families of the incomplete H-functions and the incomplete $\overline{\mathrm{H}}$-functions and associated integral transforms and operators of fractional calculus with applications. Rus. J. Math. Phys. 25: 116-138. https://doi.org/10.1134/S1061920818010119

[3] Jangid K, Bhatter S, Meena S, Baleanu D and Purohit SD (2020). Some fractional calculus findings associated with the incomplete I-functions. Adv. Differ. Equ. 2020: 265. https://doi.org/10.1186/s13662020-02725-7 
[4] Bansal MK, Kumar D, Singh J and Nisar KS (2020). On the solutions of a class of integral equations pertaining to incomplete $\mathrm{H}$-function and incomplete $\overline{\mathrm{H}}$-function. Mathematics 8(5): 819. https://doi.org/10.3390/math8050819

[5] Bansal MK, Kumar D, Nisar KS and Singh J (2020). Certain fractional calculus and integral transform results of incomplete $\aleph$-functions with applications. Math. Methods Appl. Sci. 43(8): 5602-5614. https://doi.org/10.1002/mma.6299

[6] Bansal MK, Kumar D, Singh J, Tassaddiq A and Nisar KS (2020). Some new results for the SrivastavaLuo-Raina M-transform pertaining to the incomplete H-functions[J]. AIMS Math. 5(1): 717-722. https://doi.org/10.3934/math2020048

[7] Bansal MK, Kumar D, Khan I, Singh J and Nisar KS (2019). Certain unified integrals associated with product of M-series and incomplete H-functions. Mathematics 7(12): 1191. https://doi.org/10.3390/math7121191

[8] Jangid K, Bhatter S, Meena S, Baleanu D, Al Qurashi M and Purohit SD (2020). Some fractional calculus findings associated with the incomplete I-functions. Adv. Differ Equ. 2020, 265. https://doi.org/10.1186/s13662-020-02725-7

[9] Meena S, Bhatter S, Jangid K and Purohit SD (2020). Certain integral transforms concerning the product of family of polynomials and generalized incomplete functions. Moroccan J. of Pure and Appl. Anal. (MJPAA) 6(2), 243-254. http://dx.doi.org/10.2478/mjpaa-2020-0019

[10] Baleanu D, Jangid NK, Joshi S and Purohit SD (2020). The pathway fractional integrals of incomplete I-functions. Int. J. Appl. Comput. Math..6(5), 151, 8 pp. https://doi.org/10.1007/s40819-020-00902-6

[11] Jangid NK, Joshi S, Jangid K, Araci S and Purohit SD (2021). Fractional calculus operators applied to the functions involving the product of Srivastava polynomials and incomplete I-functions. Adv. Stud. Contemp. Math., Kyungshang. 31(2), 243-258. http://dx.doi.org/10.17777/ascm2021.31.2.243

[12] Rahman G, Saboor A, Anjum Z, Nisar KS and Abdeljawad T (2020). An Extension of the Mittag-Leffler function and its associated properties. Adv. Math. Phys. 8. https://doi.org/10.1155/2020/5792853

[13] Ali RS, Mubeen S, Nayab I, Araci S, Rahman G and Nisar KS (2020). Some fractional operators with the generalized Bessel-Maitland function. Dis. Dyn. Nat. and Soc. 15. https://doi.org/10.1155/2020/1378457

[14] Rahman G, Nisar KS and Arshad M (2020). A new extension and applications of Caputo fractional derivative operator. Analysis 1. https://doi.org/10.1515/anly-2019-0005

[15] Kilbas AA, Srivastava HM and Trujillo JJ (2006). "Theory and Applications of Fractional Differential Equations". North-Holland Math Stud, Elsevier, Amsterdam, 204.

[16] Osler TJ (1972). A further extension of the Leibniz rule to fractional derivatives and its relation to Parseval's formula. SIAM J. Math. Anal. 3(1): 1-16. https://doi.org/10.1137/0503001

[17] Meena S, Bhatter S, Jangid K and Purohit SD (2020). Certain expansion formulae of incomplete Hfunctions associated with leibniz rule. TWMS. J. App. and Eng. Math. (accepted). 